\title{
CONVEX FUNCTIONS
}

E. F. BECKENBACH

1. A problem of Cauchy. In 1821, Cauchy [19] ${ }^{1}$ proposed and solved the problem of determining the class of continuous real functions $f(x)$ which satisfy the equation

$$
f\left(x_{1}\right)+f\left(x_{2}\right)=f\left(x_{1}+x_{2}\right)
$$

for all real $x_{1}$ and $x_{2}$. I think you would enjoy reading Cauchy's elegant treatment of this simple problem. But possibly you would enjoy more solving it yourself, or seeing to what considerations you are led if you omit the hypothesis of continuity.

The discontinuous solutions of (1) have been studied extensively. I have mentioned this equation because any solution of it satisfies

$$
\begin{aligned}
f\left(\frac{x_{1}+x_{2}}{2}\right) & =f\left(\frac{x_{1}}{2}\right)+f\left(\frac{x_{2}}{2}\right) \\
& =\frac{1}{2}\left\{\left[f\left(\frac{x_{1}}{2}\right)+f\left(\frac{x_{1}}{2}\right)\right]+\left[f\left(\frac{x_{2}}{2}\right)+f\left(\frac{x_{2}}{2}\right)\right]\right. \\
& =\frac{1}{2}\left[f\left(x_{1}\right)+f\left(x_{2}\right)\right],
\end{aligned}
$$

and therefore satisfies

$$
f\left(\frac{x_{1}+x_{2}}{2}\right) \leqq \frac{1}{2}\left[f\left(x_{1}\right)+f\left(x_{2}\right)\right],
$$

an inequality with which we shall be especially concerned.

2. Definition of convex function. A real function $f(x)$, defined in the interval $a<x<b$, is said to be convex provided that for all $x_{1}$ and $x_{2}$, with $a<x_{1}<x_{2}<b$, and for all positive $q_{1}$ and $q_{2}$ satisfying $q_{1}+q_{2}$ $=1$, we have

$$
f\left(q_{1} x_{1}+q_{2} x_{2}\right) \leqq q_{1} f\left(x_{1}\right)+q_{2} f\left(x_{2}\right) .
$$

A convex function necessarily is continuous for $a<x<b$.

Geometrically, the condition of convexity is that each arc of the

An address delivered before the Annual Meeting of the Society in Athens, Georgia, on December 30,1947, by invitation of the Committee to Select Hour Speakers for Annual and Summer Meetings; received by the editors January 14, 1948.

${ }^{1}$ Numbers in brackets refer to the references cited at the end of the paper. 
curve $y=f(x)$ lie nowhere above the chord joining the end points of the arc.

If $f^{\prime \prime}(x)$ exists at each point of the interval, then a necessary and sufficient condition that $f(x)$ be convex there is that we have

$$
f^{\prime \prime}(x) \geqq 0 \quad(a<x<b) .
$$

If the strict inequality in (3) holds throughout, we say that $f(x)$ is strictly convex. The terminology varies, however, and some authors use the terms "non-concave" and "convex" in place of "convex" and "strictly convex," respectively.

Similar definitions hold for concave functions. Briefly, $f(x)$ is concave if and only if $-f(x)$ is convex.

3. Elementary properties and examples. Clearly if $f(x)$ and $g(x)$ are convex functions in the interval $a<x<b$, then $f(x)+g(x)$ and $\max [f(x), g(x)]$ are convex there, as is $c f(x)$ for non-negative constants $c$.

The limit of a convergent sequence of convex functions is convex; also, if it is finite, so is the upper envelope of a family of convex functions.

A convex function has a left-hand derivative and a right-hand derivative at each point of $(a, b)$. The right-hand derivative is not less than the left-hand derivative, and both are nondecreasing functions of $x$. It follows that these derivatives are equal except at most at the point of a denumerable set of points.

If for fixed $x_{1}$ and $x_{2}$ in $(a, b)$ the sign of equality in (3) holds at a single interior point of the subinterval $\left(x_{1}, x_{2}\right)$, then the sign of equality holds throughout $\left(x_{1}, x_{2}\right)$.

In the part of $(a, b)$ outside the subinterval $\left(x_{1}, x_{2}\right)$, the graph of $y=f(x)$ lies nowhere below the line through $\left[x_{1}, f\left(x_{1}\right)\right]$ and $\left[x_{2}, f\left(x_{2}\right)\right]$.

The function $|x-a|$ is convex, its graph being $V$-shaped; therefore, for instance, so is the function

$$
g(x) \equiv 2|x|+|x-1|+|x-2|,
$$

the continuous graph of which consists of a succession of line-segments. Again, for the above function $g(x)$, the function max $\left[x^{2}, g(x)\right]$ is convex.

The differentiation test shows that for $x>0$ the functions $x \log x$ and $\log 1 / x$ are convex.

4. Early history of convex functions. Convex functions were first defined and systematically studied by J. L. W. V. Jensen $[34,35]$ in 1905 , who adopted (2) as defining inequality. We shall say that a 
function satisfying (2) for all $x_{1}$ and $x_{2}$ in $(a, b)$ is convex in the sense of Jensen, or briefly convex $(J)$. For recognizing the importance of the class of convex functions and signalizing it, Jensen deserves great credit.

Of course mathematicians were able before 1905 to recognize and utilize conditions under which the graph of a function turns its convexity downward.

Thus, as Jensen noted in an addition to his cited paper [35], in 1889 Hölder [32] had obtained the fundamental inequality

$$
f\left(\sum_{j=1}^{m} q_{j} x_{j}\right) \leqq \sum_{j=1}^{m} q_{j} f\left(x_{j}\right) \quad\left(a<x_{j}<b, q_{j}>0, \quad \sum_{j=1}^{m} q_{j}=1\right),
$$

for the subclass of convex functions $f(x)$ for which $f^{\prime \prime}(x)$ is continuous in $(a, b)$. The inequality (4) expresses the relation that the function value at the weighted average of the $x_{j}$ 's is not greater than the weighted average of the function values at the $x_{j}$ 's.

Stolz [68] in 1893 showed that if $f(x)$ is continuous and satisfies (2), then $f(x)$ has a left-hand derivative and a right-hand derivative at each point of $(a, b)$.

Also in 1893, Hadamard [24] showed that if $f(x)$ has an increasing derivative (so that $f(x)$ is convex), then for $a<x_{1}<x_{2}<b$ we have

$$
f\left(\frac{x_{1}+x_{2}}{2}\right)<\frac{1}{x_{2}-x_{1}} \int_{x_{1}}^{x_{2}} f(x) d x .
$$

In 1896, Hadamard [25] announced the result which Landau [40] later glamorized by designating it the Three Circles Theorem: If $f(z)$ is an analytic function of the complex variable $z$ in the annulus $a<|z|<b$, and $M(r ;|f|)$ denotes the maximum of $|f(z)|$ on the circle $|z|=r$, then the graph of $\log M(r ;|f|)$ as a function of $\log r$ turns its convexity downward. That is, $\log M$ is a convex function of $\log r$.

In 1897 and 1898, Hadamard [26, 27] observed that for a surface $S$ given in geodesic representation,

$$
d s^{2}=d u^{2}+\mu^{2} d v^{2} \quad(\mu \geqq 0),
$$

the Gaussian, or total, curvature $K$ is given at points where $\mu \neq 0$ by

$$
K=-\frac{1}{\mu} \frac{\partial^{2} \mu}{\partial u^{2}}
$$

so that if $K$ is of one sign over all of $S$, then $\partial^{2} \mu / \partial u^{2}$ is of the opposite sign. Thus if $S$ is a surface of negative curvature, then $\mu\left(u, v_{0}\right)$ is a convex function of $u$. 
Let

$$
\sum_{j, k=0}^{\infty} a_{j, k} z^{j} w^{k}
$$

be a power series in two complex variables, which is convergent for a pair of values $z_{0}, w_{0}$ neither of which is zero. That is, every simple series formed from the terms of (6) converges for $z=z_{0}, w=w_{0}$. Then (6) converges absolutely for all $z$, $w$ satisfying $|z|<\left|z_{0}\right|,|w|<\left|w_{0}\right|$. A pair of positive numbers $r$ and $\rho$ such that the series converges for $|z|<r$ and $|w|<\rho$ simultaneously, but diverges for $|z|>r$ and $|w|>\rho$ simultaneously, is called $a$ pair of associated radii of convergence. Thus for

$$
\sum_{j=0}^{\infty} z^{i} w^{j}=\frac{1}{1-z w}
$$

clearly $r \rho=1$, so that $1 / \rho=r$ and $\log 1 / \rho=\log r$.

In 1902, Fabry [22] showed that if $r$ and $\rho$ are associated radii of convergence, then $\log 1 / \rho$ is a convex function of $\log r$, as illustrated in the above example. Faber [21] and Hartogs [31] showed conversely that if $r$ and $\rho$ are positive variables such that $\log 1 / \rho$ is a convex function of $\log r$, then there exist series of the form (6) for which $r$ and $\rho$ are associated radii of convergence.

Minkowski [44] in 1903 studied convex bodies by means of Stïtzfunktionen, which are particular convex functions of more than one independent variable.

Possibly the convexity of some significant convex functions which were studied before 1905 was recognized though not mentioned. Let $f(z)$ be analytic for $|z| \leqq r$, with a zero of order $m \geqq 0$ at $z=0$, so that

$$
f(z) \equiv z^{m} g(z),
$$

where $g(0) \neq 0$, and let the moduli of the zeros of $f(z)$ in $0<|z|<r$ be $r_{1}, r_{2}, \cdots, r_{n}$, with $0<r_{1} \leqq r_{2} \leqq \cdots \leqq r_{n}$. Then we have the familiar formula

$$
\frac{1}{2 \pi} \int_{0}^{2 \pi} \log \left|f\left(r e^{i \theta}\right)\right| d \theta=\log \frac{|g(0)| r^{m+n}}{r_{1} r_{2} \cdots r_{n}}
$$

which can be written as

$$
\mathfrak{M}_{0}(r ;|f|) \equiv \exp \frac{1}{2 \pi} \int_{0}^{2 \pi} \log \left|f\left(r e^{i \theta}\right)\right| d \theta=\frac{|g(0)| r^{m+n}}{r_{1} r_{2} \cdots r_{n}} .
$$

Thus as $r$ increases from $r=0$, the exponent $m+n$ increases by in- 
tegral amounts at the moduli of the zeros of $f(z)$, and the geometric mean $\mathfrak{M}_{0}(r ;|f|)$ is, by successive intervals some of which might be degenerate, constant out to the modulus of the first zero of $f(z)$, then a linear function of $r$, then a quadratic, and so on.

Clearly it follows from (7) and (8) that $\log \mathfrak{M}_{0}(r ;|f|)$ is a convex function of $\log r$; this is a limting case of a theorem established by Hardy [29] in 1914. Actually it follows also from (8) that $\mathfrak{M}_{0}(r ;|f|)$ is a convex function of $r$ [6]. However, the discoverer of (7) remarked in this connection only that the left-hand member of (7) is a nondecreasing function of $r$. Established in 1898, (7) is known as Jensen's formula [33].

5. Functions convex in the sense of Jensen. If $f(x)$ is convex, then $f(x)$ satisfies (2) as special case of (3); that is, if $f(x)$ is convex, then $f(x)$ is convex in the sense of Jensen, or convex $(J)$. Conversely, if $f(x)$ is continuous and convex $(J)$, then $f(x)$ is convex. But, though a convex function must be continuous, a function which is convex $(J)$ is not necessarily continuous.

As Hamel [28] showed in 1905, the existence of discontinuous functions $f(x)$ which satisfy (1) is readily established by means of a Hamel basis $B$ for the real numbers.

A Hamel basis $B$ for the real numbers is a set of real numbers $b$, the elements of $B$, such that each real $x \neq 0$ has a unique representation of the form

$$
x=\sum_{j=1}^{n(x)} r_{j} b_{j}
$$

where $n(x)$ is finite, the $r_{j}$ are rational numbers with $r_{j} \neq 0$, and the $b_{j}$ are elements of $B$.

The general solution of (1) is given by

$$
f(x)=f\left(\sum_{j=1}^{n(x)} r_{j} b_{j}\right)=\sum_{j=1}^{n(x)} r_{j} f\left(b_{j}\right) \quad(x \neq 0 ; f(0)=0),
$$

where $f(b)$ denotes an arbitrary function on $B$.

Since the set of real numbers is non-denumerable, and the $r_{j}$ are rational, the set of elements of $B$ must be non-denumerable, and therefore there must be an accumulation point $p_{0}$ of $B$ in $B$. If, for instance, we define $f(b)$ to be 0 on $B$ except at the accumulation point $p_{0}$, and take $f\left(p_{0}\right)=1$, then $f(x)$ necessarily is discontinuous at $p_{0}$.

To obtain a discontinuous function which is strictly convex $(J)$, we might then, for instance, add $x^{2}$ to the above function $f(x)$; or we might take the function 


$$
f^{*}(x) \equiv \max \left[x^{2}, f(x)+x^{2}\right],
$$

which is bounded from below by $y=x^{2}$.

6. Generalizations of convex functions. In 1908, Phragmén and Lindelö [51] showed that if $f(z)$ is an entire function of finite order $\rho$, then the function

$$
h(\theta) \equiv \limsup _{r \rightarrow \infty} \frac{\log \left|f\left(r e^{i \theta}\right)\right|}{r^{\rho}}
$$

has the following property: If $0<\theta_{2}-\theta_{1}<\pi / \rho$, and $H(\theta)$ is the function of the form

$$
A \cos \rho \theta+B \sin \rho \theta
$$

which coincides with $h(\theta)$ at $\theta_{1}$ and at $\theta_{2}$, then for $\theta_{1}<\theta<\theta_{2}$ we have

$$
h(\theta) \leqq H(\theta) \text {. }
$$

Accordingly, $h(\theta)$ is said to be a sub-trigonometric function. Pólya [53] showed that sub-trigonometric functions have certain differential properties in common with convex functions, and Valiron [69] extended the analysis to functions $f(x)$ dominated by functions of the form

$$
A \phi(x)+B \psi(x)
$$

for suitably restricted functions $\phi(x)$ and $\psi(x)$.

More generally, let $\{F(x)\}$ be a family of continuous functions $F(x)$ defined in an interval $a<x<b$, such that for given $P_{1}:\left(x_{1}, y_{1}\right)$ and $P_{2}:\left(x_{2}, y_{2}\right)$, with $a<x_{1}<x_{2}<b$, there is a unique member of $\{F(x)\}$ through $P_{1}$ and $P_{2}$. Functions $f(x)$ dominated by $\{F(x)\}$ might be said [1] to be convex relative to $\{F(x)\}$. With Bing [5], we have defined functions which are convex $(J)$ relative to $\{F(x)\}$.

There are families $\{F(x)\}$ which are not topologically equivalent to the family $\{L(x)\}$ of all non-vertical line-segments terminating on $x=a, x=b$. And there are families $\{F(x)\}$ such that $f(x)$ might be convex relative to $\{F(x)\}$ yet nowhere differentiable. But many properties of convex functions, particularly those concerning measure, do hold for these general functions.

In terms of divided differences of order $n$, Popoviciu [55] has defined convex functions of order $n$. If a convex function $f(x)$ of order $n$ and a polynomial $p(x)$ of degree $n$ (or less) have equal values for $n+1$ values of $x$, then alternately $f(x) \leqq p(x)$ and $f(x) \geqq p(x)$ in the successive subintervals bounded by these $n+1$ values of $x$.

My colleague Drandell [20] is combining the notions of functions 
convex relative to $\{F(x)\}$ and of convex functions of order $n$. Added in proof: Shortly before the delivery of this address, results in this same program were announced by Tornheim in Bull. Amer. Math. Soc. vol. 53 (1947) pp. 1119-1120.

A function $f(x, y)$ is said to be doubly convex provided that, in its domain of definition, $f(x, y)$ is a convex function of $x$ for each $y$, and a convex function of $y$ for each $x$. Thus $f(x, y) \equiv x y$ is doubly convex Of several additional generalizations of convex functions $f(x)$, we shall later consider two additional generalizations to functions of two (or more) independent variables; these are (i) convex functions of $P:(x, y)$, and (ii) subharmonic functions.

To indicate the possibility of yet another generalization, we recall the inequality of Steiner [42]: For two continuous surfaces

$$
S_{1}: \quad z=z_{1}(x, y), \quad S_{2}: \quad z=z_{2}(x, y), \quad(x, y) \text { in } R,
$$

where $R$ is a Jordan region, let $S$ denote the surface

$$
S: \quad z=\left[z_{1}(x, y)+z_{2}(x, y)\right] / 2 .
$$

If $A(S)$ denotes the area of $S$, then we have

$$
A(S) \leqq\left[A\left(S_{1}\right)+A\left(S_{2}\right)\right] / 2 .
$$

7. Measure and connectedness. Investigations of the discontinuous solutions of (1) and (2) have been concerned particularly with properties of measure and connectedness.

Jensen [35] proved that if $f(x)$ satisfies (2) and is bounded from above, then $f(x)$ is continuous. This result has been extended by Bernstein and Doetsch [9], Blumberg [11], Sierpenski [67], and Ostrowski [49].

If $f(x)$ is convex $(J)$ and is not continuous, then $f(x)$ cannot be bounded on any subinterval or even on a set of positive measure; $f(x)$ cannot be a measurable function; and either the set $M$ of points of the graph of $y=f(x)$ is dense in the entire strip $a<x<b,-\infty<y<+\infty$, or there is a continuous convex function $\phi(x)$ such that the set $M$ lies in $a<x<b, \phi(x)<y<+\infty$, and is dense there. For example, the graph of the above function $f^{*}(x)$ is dense in the part of the plane above $y=x^{2}$. For any circular disc $D$ such that $M$ is dense in $D$, the $x$-projection of the part of $M$ in $D$ has zero interior measure and positive exterior measure.

It has been shown by Jones [36] that the graphs $y=f(x)$ of the discontinuous solutions of (1) serve to illustrate relatively easily certain weird topological properties of connected sets. Thus there are discontinuous solutions of (1) for which the graphs are connected, and 
others for which the graphs are totally disconnected. But even though the graph might be connected, it must be punctiform; that is, it can contain no nondegenerate continuum.

We shall return to considerations of measure and connectedness in discussing convex sets.

8. Convex sets. A set $S$ of points in the plane or in space is convex provided that for each pair of points $P_{1}$ and $P_{2}$ in $S$, the entire linesegment $P_{1} P_{2}$ is contained in $S[44,13]$.

A function $f(P)$, defined on a convex set $S$, is said to be convex provided that for each pair of points $P_{1}$ and $P_{2}$ in $S$, and for all positive $q_{1}$ and $q_{2}$ satisfying $q_{1}+q_{2}=1$, we have

$$
f\left(q_{1} P_{1}+q_{2} P_{2}\right) \leqq q_{1} f\left(P_{1}\right)+q_{2} f\left(P_{2}\right),
$$

where $q_{1} P_{1}+q_{2} P_{2}$ has its obvious meaning.

To illustrate the connection between convex functions and convex sets, I shall state a simple theorem suggested to me by Newburgh, one of my colleagues; I think you might enjoy supplying the few lines necessary for its proof.

Theorem. For a closed set $S$ and a variable point $P$, let

$$
d(P ; S) \equiv \underset{Q \text { on } S}{\min } \operatorname{distance}(P, Q) .
$$

$A$ necessary and sufficient condition that $S$ be a convex set is that $d(P ; S)$ be a convex function of $P$.

Two of my colleagues, Green and Gustin [23], have defined subconvex sets $S$ of points as follows. Let $\lambda$ denote a non-null set of points in the interval $0<x<1$. Then the set $S$ is sub-convex, or convex relative to $\lambda$, provided that for each pair of points $P_{1}$ and $P_{2}$ of $S$, all the points of the image of $\lambda$ under the homothetic mapping of the line-segment $0<x<1$ on the line-segment $P_{1} P_{2}$ are contained in $S$.

If $S$ is convex, then $S$ is convex relative to any $\lambda$.

Green and Gustin have found that many properties concerning measure and connectedness of solutions of (1) have analogues in the theory of sub-convex sets. Thus if a set $S$, convex relative to $\lambda$, contains a nonlinear continuum, or is of positive interior measure, then $S$ is nearly convex; that is, there is a convex set $T$ such that $S$ is contained in $T$, and $S$ coincides with $T$ except for the possible omission of part of the boundary of $T$. Again, if a set $S$, convex relative to $\lambda$, contains a nonlinear connected set, then $S$ is connected.

9. Inequalities. Jensen gave the study of (algebraic) inequalities as 
principal object of his investigation of convex functions. He showed that the basic inequality (4) holds provided $f(x)$ is convex $(J)$ and the $q_{j}$ are rational, whence it follows that if $f(x)$ is continuous and convex $(J)$ then the hypothesis that the $q_{j}$ are rational can be dropped.

The inequality (4) has been generalized by McShane [43].

Applications of (4) to certain convex functions yield familiar algebraic inequalities, including the three which are usually taken to be fundamental: the inequality between the geometric and arithmetic means, the inequality of Hölder, and the inequality of Minkowski.

Thus from the convexity of $-\log x$ for $x>0$ we obtain [54]

$$
-\log \left(\sum_{j=1}^{n} q_{j} a_{j}\right) \leqq-\sum_{j=1}^{n} q_{j} \log a_{j}=-\log \prod_{j=1}^{n} a_{j}^{q_{j}} \quad\left(a_{j}>0\right)
$$

or

$$
\prod_{j=1}^{n} a_{j}^{q_{j}} \leqq \sum_{j=1}^{n} q_{j} a_{j}
$$

which is the inequality between the geometric and arithmetic means.

Hölder's inequality can be obtained from (9), and Minkowski's inequality follows from Hölder's [65].

The inequality between the geometric and arithmetic means is a special case of a more general inequality [10]. We define the mean of order $t$ for positive values $(a) \equiv\left(a_{1}, a_{2}, \cdots, a_{n}\right), n \geqq 2$, and positive weights $(q) \equiv\left(q_{1}, q_{2}, \cdots, q_{n}\right)$ with $\sum_{j=1}^{n} q_{j}=1$, by

$$
\mathfrak{M}_{t}(a ; q) \equiv\left(\sum_{j=1}^{n} q_{j} a_{j}^{t}\right)^{1 / t} \quad(-\infty<t<0 \text { or } 0<t<+\infty),
$$

and

$$
\mathfrak{N}_{0}(a ; q) \equiv \prod_{j=1}^{n} a_{j}^{q_{j}}, \quad \mathfrak{M}_{-\infty}(a ; q) \equiv \min (a), \quad \mathfrak{M}_{+\infty}(a ; q) \equiv \max (a) .
$$

Thus for $t=-1,0,1$, and $2, \mathfrak{M}_{t}$ is respectively the harmonic mean, the geometric mean, the arithmetic mean, and the "root-meansquare."

For positive continuous functions $f(x)$ and $q(x)$ in $(a, b)$, with $\int_{a}^{b} q(x) d x=1$, the integral analogue of $\mathfrak{M}_{t}(a ; q)$ is given by

$$
\begin{aligned}
& \mathfrak{M}_{t}(f ; q) \equiv\left\{\int_{a}^{b} q(x)[f(x)] t d x\right\}^{1 / t} \\
&(-\infty<t<0 \text { or } 0<t<+\infty),
\end{aligned}
$$


and

$$
\begin{gathered}
\mathfrak{M}_{-\infty}(f ; q) \equiv \min f(x), \quad \mathfrak{M}_{+\infty}(f ; q) \equiv \max f(x), \\
\mathfrak{M}_{0}(f ; q) \equiv \exp \int_{a}^{b} q(x) \log f(x) d x .
\end{gathered}
$$

The function $\mathfrak{M}_{t}(a ; q)$ is a continuous function of $t$ for $-\infty \leqq t \leqq$ $+\infty$.

The general inequality to which we have referred is

$$
\mathfrak{M}_{s}(a ; q) \leqq \mathfrak{M}_{t}(a ; q) \quad(-\infty \leqq s<t \leqq+\infty),
$$

where the sign of equality holds if and only if all the $a_{j}$ are equal.

The sums of order $t$, defined by

$$
\mathfrak{S}_{t}(a) \equiv\left(\sum_{j=1}^{n} a_{j}^{t}\right)^{1 / t} \quad(-\infty<t<0 \text { or } 0<t<+\infty),
$$

behave quite differently $[56,35]$, with

$$
\mathfrak{\varsigma}_{8}(a)>\mathfrak{\Im}_{t}(a)
$$

provided $s$ and $t$ are both positive or both negative. Further,

$$
\begin{aligned}
& \lim _{t \rightarrow-\infty} \mathfrak{S}_{t}(a)=\min (a), \quad \lim _{t \rightarrow 0, t<0} \mathfrak{S}_{t}(a)=0, \\
& \lim _{t \rightarrow 0, t>0} \mathfrak{S}_{t}(a)=+\infty, \quad \lim _{t \rightarrow+\infty} \mathfrak{S}_{t}(a)=\max (a) .
\end{aligned}
$$

The inequality (10) is sometimes [30] called Jensen's inequality, though I prefer to reserve this term for (4). Actually, neither inequality originated with Jensen.

10. Further study of known inequalities. The inequalities which we have been discussing express the fact that certain functions are nondecreasing or nonincreasing functions of certain parameters. The inequalities do not, however, tell how the functions increase or decrease.

Thus since the graph of $y=\mathfrak{M}_{t}(a ; q)$ has two horizontal asymptotes, it must have at least one inflection point [48]. Does it necessarily have exactly one inflection point, so that $\mathfrak{M}_{t}(a ; q)$ must be a convexoconcave function, or might there be several inflection points? There are examples in the literature [48] showing that at $t=0, d^{2} \mathfrak{M N}_{t} / d t^{2}$ might be positive, negative, or zero, so that if there were necessarily only one inflection point it would seem to be an elusive one.

It is known [30] that $\log \left\{\left[\mathfrak{M}_{t}(a ; q)\right]^{t}\right\}$ is a convex function of $t$, as is $\log \left\{\left[\Im_{t}(a)\right]^{t}\right\}$; it follows that $\left[\mathfrak{M}_{t}(a ; q)\right]^{t}$ and $\left[\Im_{t}(a)\right]^{t}$ are con- 
vex functions of $t$, since the convexity of the logarithm of a function implies the convexity of the function itself.

It is known also [39] that $\log \mathfrak{M}_{t}(a ; q)$ and $\log \mathfrak{S}_{t}(a)$ are convex functions of $1 / t$ for $t>0$, and concave functions of $1 / t$ for $t<0$.

Recently Shniad [66], one of my colleagues, has shown that $\mathfrak{M}_{t}(a ; q)$ is not necessarily a convexo-concave function of $t$. Explicitly, for the function

$$
\mathfrak{M}_{t}(a ; q)=\left(\frac{1}{10} e^{t}+\frac{8}{10} e^{2 t}+\frac{1}{10} e^{3 t}\right)^{1 / t},
$$

the second derivative is positive at $t=-2$, negative at $t=-1$, positive at $t=0$, and negative at $t=4$.

On the other hand, the ingenious analysis which led Shniad to consider the above example yielded the positive result that for any given $(a ; q)$ there exist (finite) values $t_{j}=t_{j}(a ; q), j=1,2$, such that log $\mathfrak{M A}_{t}(a ; q)$ is a convex function of $t$ for $t<t_{1}$, and a concave function of $t$ for $t>t_{2}$. Consequently, $\mathfrak{M}_{t}(a ; q)$ also must be a convex function of $t$ for $t<t_{1}$.

As for $\mathfrak{S}_{t}(a)$, Bonnesen [12] has shown that this is a convex function of $t$ for $t>1$. Later, by a different method, I obtained [3] the same result for $t>0$.

Several results which I shall mention later also involve the improvement of known inequalities by the establishment of convexity properties.

11. A hierarchy of convexity conditions. I have remarked that the convexity of the logarithm of a positive function implies the convexity of the function itself. This result holds as an instance of the following continuous hierarchy of convexity conditions.

Let $C_{\alpha}$ denote the class of positive functions $p(x)$ defined in $(a, b)$ such that the function

$$
\operatorname{sg}(\alpha)[p(x)]^{\alpha} \quad(\alpha \neq 0),
$$

or

$$
\log p(x) \quad(\alpha=0),
$$

is a convex function of $x$ in $(a, b)$, where $\operatorname{sg}(\alpha)=-1$ for $\alpha<0$ and $\operatorname{sg}(\alpha)=+1$ for $\alpha>0$.

Then $p(x)$ is a member of $C_{\alpha}$ if and only if $p(x)$ is a member of $C_{\beta}$ for all $\beta>\alpha[57]$.

The class $C_{0}$ of functions whose logarithms are convex is particularly amenable to analysis, in that the class is closed both under addi- 
tion and under multiplication [7]. The class also is particularly important, in that several physically significant functions are members of $C_{0}$.

Thus in complex variable theory there are several functions, some of which we have mentioned already, which are members of $C_{0}$ relative to $\log r$ as independent variable:

For associated radii of convergence, $\log 1 / \rho$ is a convex function of $\log r$.

In the theory of meromorphic functions, the Nevanlinna function

$$
T(r) \equiv \frac{1}{2 \pi} \int_{0}^{2 \pi} \log ^{+}\left|f\left(r e^{i \theta}\right)\right| d \theta+\log \frac{r^{n}}{r_{1} r_{2} \cdots r_{n}}
$$

involves logarithms in such a way that it seems natural to consider the function

$$
U(r) \equiv \exp T(r) .
$$

A fundamental result of Nevanlinna [47] is that $\log U(r)$ is a convex function of $\log r$.

The Hadamard Three Circles Theorem expresses the convexity of

$$
\log M(r ;|f|) \equiv \log \mathfrak{M}_{+\infty}(r ;|f|)
$$

as function of $\log r$.

For the geometric mean $\mathfrak{M}_{0}(r ;|f|)$ we have pointed out the result that $\log \mathfrak{M}_{0}(r ;|f|)$ is a convex function of $\log r$.

But we have indicated also that $\mathfrak{M}_{0}(r ;|f|)$ satisfies the additional condition that $\mathfrak{M}_{0}(r ;|f|)$ itself is a convex function of $r$ itself.

Which is the stronger convexity condition on a positive function $p(r)$, the condition that $\log p(r)$ be a convex function of $\log r$, or the condition that $p(r)$ be a convex function of $r$ ? The answer is that neither implies the other, for each can hold in the absence of the other [6].

We shall pursue this matter further in the next section, in which we discuss a continuum of functions whose logarithms are convex functions of $\log r$.

12. Hardy's theorem. In $\mathfrak{M}_{t}(r ;|f|), 0 \leqq t \leqq+\infty$, we have a continuum of functions of class $C_{0}$, in accordance with the following theorem of Hardy [29] to which we referred in $\$ 4$.

HARDY'S THEOREM. Let $f(z)$ be an analytic function of the complex variable $z$ in $|z|<1$. Then $\log \mathfrak{M}_{t}(r ;|f|)$ is a (nondecreasing) convex function of $r$ for any non-negative value $t$. 
We already have considered the limiting cases $t=0$ and $t=+\infty$.

The question arises as to whether or not $\mathfrak{M}_{t}(r ;|f|)$ is necessarily a convex function of $r$ for $0 \leqq t \leqq+\infty$.

Gustin, Shniad, and I currently are investigating the above question, and have obtained the following results.

For any function $f(z)$ analytic in $|z|<1$, and for any $t$ satisfying $0 \leqq t \leqq+\infty$, let $\rho(t ;|f|)$ denote the least upper bound of values $\rho$ such that $\mathfrak{M}_{t}(r ;|f|)$ is convex for $0<r<\rho$. Let $\rho(t)$ denote the greatest lower bound of $\rho(t ;|f|)$ for $f$ ranging over the class of functions analytic in $|z|<1$. Then $0 \leqq \rho(t) \leqq \rho(t ;|f|) \leqq 1$, and we have indicated that $\rho(0)=1$.

For the function

$$
F(z) \equiv \frac{z+a}{1+a z} \quad(0<a<1,|z|<1),
$$

the maximum-value function

$$
M(r ;|F|) \equiv \mathfrak{M}_{+\infty}(r ;|F|)
$$

is strictly concave for $0<r<1$. Accordingly, we have $\rho(+\infty ;|F|)=0$, whence $\rho(+\infty)=0$.

Also, since $\mathfrak{M}_{t} \rightarrow \mathfrak{M}_{+\infty}$ as $t \rightarrow+\infty$, and since the limit of a convergent sequence of convex functions is convex, it follows that

$$
\lim _{t \rightarrow+\infty} \rho(t)=0 .
$$

If $f(z)$ has at most one zero in $|z|<1$, then we have the result that

$$
\rho(t ;|f|)=1 \quad(0 \leqq t \leqq 2) ;
$$

if $f(z)$ has at most two zeros, then

$$
\rho(t ;|f|)=1
$$$$
(0 \leqq t \leqq 1) ;
$$

and there are similar results for any number of zeros.

Irrespective of the number of zeros of $f(z)$, we have

$$
\rho(t)=1, \quad(t=2 / k ; k=1,2, \cdots, n, \cdots) .
$$

The previously noted result $\rho(0)=1$ follows by a limiting process, with $k \rightarrow+\infty$.

Whether or not there are other values of $t$ for which $\rho(t)=1$, or any values of $t$ for which $0<\rho(t)<1$, we do not now know.

Added in proof. Shortly after the delivery of this address, Shniad showed that we have $\rho(t)<1$ for all $t>8$.

As an application, we recall the known result that the length $l(r)$ 
of the image of $|z|=r$ under the transformation $w=f(z)$ is a nondecreasing function of $r$ : if $0<r_{1}<r_{2}<1$, then $l\left(r_{1}\right) \leqq l\left(r_{2}\right)$. It now can be shown that $l(r)$ is a convex function of $r$.

One of the most attractive results concerning $\rho(t ;|f|)$ was obtained by Shniad as a consequence of the theorem of Hardy: namely, we have

$$
\rho(t ;|f(z)-f(0)|)=1 \quad(0 \leqq t \leqq+\infty) .
$$

This result, which is an instance of a theorem of Nehari [46], involves an interesting implication relative to the lemma of Schwarz, as we shall see in the next section.

13. The lemma of Schwarz and convexity. Recalling that

$$
\mathfrak{M}_{+\infty}(r ;|f|) \equiv \max _{0 \leqq \theta<2 \pi}\left|f\left(r e^{i \theta}\right)\right|,
$$

we may state the Lemma of Schwarz as follows.

Lemma of Schwarz. Let $f(z)$ be an analytic function of the complex variable $z$ in $|z|<1$, with $f(0)=0$. If for all $r, 0<r<1$, we have

$$
\mathfrak{M}_{+\infty}(r ;|f|) \leqq 1 \text {, }
$$

then we have

$$
\mathfrak{M}_{+\infty}(r ;|f|) \leqq r \quad(0<r<1)
$$

and

$$
\left|f^{\prime}(0)\right| \leqq 1,
$$

the signs of equality holding if and only if $f(z) \equiv e^{i \alpha} z$, where $\alpha$ is a real constant.

Thus if the origin is mapped on the origin by the analytic function $w=f(z)$, and the map of the unit circle $|z|<1$ lies in the unit circle $|w|<1$, then the map of any smaller concentric circle of radius $r$ lies in the concentric circle of radius $r$, and reaches the boundary $|w|=r$ if and only if the map is a rotation.

It is known [38] that the Lemma of Schwarz extends to means of other orders. In the statement of the lemma, we have only to replace $\mathfrak{M}_{+\infty}$ throughout by $\mathfrak{M}_{t_{0}}, 0 \leqq t_{0} \leqq+\infty$. Indeed, for $0<r_{1}<r_{2}<1$, we have [38], more precisely,

$$
\frac{\mathfrak{M}_{\iota_{0}}\left(r_{1} ;|f|\right)}{\mathfrak{M}_{\iota_{0}}\left(r_{2} ;|f|\right)} \leqq \frac{r_{1}}{r_{2}} \quad\left(0 \leqq t_{0} \leqq+\infty\right) .
$$

Now (11) does not imply that the curve $y=\mathfrak{M}_{t_{0}}(r ;|f|)$ is convex, 
but only that arcs of the curve having one end point at the origin lie nowhere above the corresponding chords. However, as we pointed out in $\$ 12$, since $f(0)=0$ the curve $y=\mathfrak{M}_{t_{0}}(r ;|f|)$ actually is convex.

14. Subharmonic functions. In studying associated radii of convergence, Hartogs [31] in 1906 used as tool a real function $R(\zeta)$ of the complex variable $\zeta$, defined as follows.

Let $f(z, w)$ be analytic at the point $(\zeta, 0)$,

$$
f(z, w) \equiv \sum_{j, k=0}^{\infty} a_{j, k}(z-\zeta)^{j} w^{k}
$$

and let $r$ and $\rho=\phi(r)$ be associated radii of convergence of the series. Then by definition we have

$$
R(\zeta) \equiv \lim _{r \rightarrow 0} \phi(r)
$$

Hartogs showed that the function

$$
g(x, y) \equiv \log \frac{1}{R(\zeta)} \quad(\zeta=x+i y)
$$

is upper semi-continuous in its domain of definition $D$, and has the following property. If $D^{\prime}$ is a domain lying together with its boundary $B^{\prime}$ in $D$, and $h(x, y)$ is harmonic in $D^{\prime}$ and continuous in $D^{\prime}+B^{\prime}$, and we have $g(x, y) \leqq h(x, y)$ on $B^{\prime}$, then necessarily we have also $g(x, y)$ $\leqq h(x, y)$ throughout $D^{\prime}$.

Since a harmonic function $h(x, y)$ is by definition a solution of the partial differential equation

$$
\Delta h \equiv \frac{\partial^{2} h}{\partial x^{2}}+\frac{\partial^{2} h}{\partial y^{2}}=0,
$$

the class of harmonic functions is a generalization, to functions of two (or more) independent variables, of the class of linear functions of one variable. Accordingly, the above property of domination by harmonic functions is a generalization of the defining property of convex functions of one variable.

Harmonic functions $h(x, y)$ are characterized by the mean-value property that if the circular disc $\left(x-x_{0}\right)^{2}+\left(y-y_{0}\right)^{2} \leqq r^{2}$ is in the domain of definition, then

$$
h\left(x_{0}, y_{0}\right)=\frac{1}{2 \pi} \int_{0}^{2 \pi} h\left(x_{0}+r \cos \theta, y_{0}+r \sin \theta\right) d \theta .
$$

If the above function $g(x, y)$ is $\not=-\infty$, then $g(x, y)$ satisfies the 
mean-value inequality

$$
g\left(x_{0}, y_{0}\right) \leqq \frac{1}{2 \pi} \int_{0}^{2 \pi} g\left(x_{0}+r \cos \theta, y_{0}+r \sin \theta\right) d \theta,
$$

though Hartogs did not explicitly give this result.

Hartogs showed that if the above function $g(x, y)$ is continuous together with its partial derivatives of the first and second orders, then $g(x, y)$ satisfies the differential inequality

$$
\Delta g \equiv \frac{\partial^{2} g}{\partial x^{2}}+\frac{\partial^{2} g}{\partial y^{2}} \geqq 0 .
$$

Later Levi [41] in 1910 and Julia [37] in 1925 used functions analogous to $g(x, y)$ in the study of poles and essential singularities of analytic functions of several complex variables.

In 1922, Riesz [61] made the remarkable discovery that the theorem of Hardy, to which we have referred, and which previously had been proved only with considerable difficulty, can be established very simply from the fact that $|f(z)|^{t}$ satisfies the mean-value inequality (12).

In a series of papers starting in 1923, by Perron [50], Remak [60], Radó and Riesz [59], Wiener [72], Whitney [71], and Carathéodory [15], the solution of the Dirichlet problem in potential theory was put in very elegant form by means of functions having the property to which we have referred. Actually, as Riesz [64] pointed out in 1926 , the idea of domination by harmonic functions was involved in potential theory as early as 1887 in the sweeping-out process of Poincaré [52].

It now appeared that the class of functions involved was of intrinsic interest, because of its various applications and because of its relation to convex functions. Riesz $[62,63,64]$ defined a subharmonic function to be an upper semi-continuous function $f(x, y)$ which satisfies $-\infty \leqq f(x, y)<+\infty, f(x, y) \not \equiv-\infty$, and which is dominated by harmonic functions as described above.

To be exact, Riesz assumed the apparently but (as Evans [58] pointed out) not actually stronger condition that $f(x, y)$ is not equal to $-\infty$ on a set of points dense in the domain of definition $D$, in place of the condition that $f(x, y)$ is not identically equal to $-\infty$. Personally, for several reasons, I prefer to include the function $f(x, y) \equiv-\infty$ in the class of subharmonic functions.

Essentially, subharmonic functions were involved in the study of differential geometry by Weil [70] in 1926, when he extended the 
isoperimetric inequality

$$
a \leqq \frac{1}{4 \pi} l^{2}
$$

to surfaces of negative curvature, after Carleman [17] had shown in 1921 that (13) holds for Jordan regions of area $a$ and length of perimeter $l$ on minimal surfaces. The inequality (13) characterizes [8] surfaces of non-positive Gaussian curvature.

Thus properties of subharmonic functions now are investigated from four points of view: the study of properties of subharmonic functions for their intrinsic interest, in particular as they relate to convex functions [45]; and the study of applications in potential theory, complex variable theory, and differential geometry. The four studies are mutually stimulating.

To illustrate the first point of view, we might ask the following question: If $h(x, y)$ is harmonic, and $s(x, y)$ subharmonic, in the unit circle $x^{2}+y^{2}<1$, and $h(x, y) \equiv s(x, y)$ on $x^{2}+y^{2}=r_{0}^{2}$ for some $r_{0}$ with $0<r_{0}<1$, do we necessarily have $h(x, y) \leqq s(x, y)$ for $r_{0}^{2}<x^{2}+y^{2}<1$ ?

We shall conclude our remarks with some observations concerning differential geometry as related to convex functions, subharmonic functions, and functions of complex variables.

15. Differential geometry. Both convex functions and subharmonic functions serve as tools in the study of differential geometry.

The use of convex functions in differential geometry, which, as we mentioned, was initiated by Hadamard, has been extended to Riemannian spaces by Cartan [18].

In our local peripatetic seminar, Professor Busemann [14] recently has shown how, without use of differentiability hypotheses, the same results can be carried over to general metric spaces $S$ of nonpositive curvature: $S$ is said to be of nonpositive curvature provided each point of $S$ has a neighborhood $N$ such that the side $b c$ of any geodesic triangle $a b c$ in $N$ is at least twice as long as the (shortest) geodesic arc connecting the midpoints $b^{\prime}, c^{\prime}$ of the other two sides:

$$
\overline{b^{\prime} c^{\prime}} \leqq \overline{b c} / 2 \text {. }
$$

On the other hand, elementary calculus can be used [4] to obtain, from (5), various inequalities and convexity conditions involving length and area on surfaces of nonpositive curvature and on surfaces of non-negative curvature.

Subharmonic functions are related to differential geometry especially in accordance with the following two theorems $[7,8]$. 
THEOREM A. Three real functions $x(u, v), y(u, v), z(u, v)$, continuous in a domain $D$, are coordinate functions of a minimal surface in conformal representation, that is, the functions are harmonic and satisfy

$$
E=G=\lambda(u, v), \quad F=0,
$$

where

$$
E=x_{u}^{2}+y_{u}^{2}+z_{u}^{2}, \quad F=x_{u} x_{v}+y_{u} y_{v}+z_{u} z_{v}, \quad G=x_{v}^{2}+y_{v}^{2}+z_{v}^{2},
$$

if and only if the distance function

$$
\rho(u, v ; a, b, c) \equiv\left\{[x(u, v)-a]^{2}+[y(u, v)-b]^{2}+[z(u, v)-c]^{2}\right\}^{1 / 2}
$$

satisfies the condition that $\log \rho$ is a subharmonic function of $(u, v)$ for every choice of the real constants $a, b, c$.

Theorem B. A necessary and sufficient condition that a surface $S$,

$$
S: \quad x=x(u, v), \quad y=y(u, v), \quad z=z(u, v),
$$

given in conformal representation,

$$
E=G=\lambda(u, v), \quad F=0,
$$

be a surface of nonpositive Gaussian curvature is that $\log \lambda$ be a subharmonic function of $(u, v)$.

Briefly, in accordance with Theorem A the principle of the maximum for the moduli $|f(z)|$ of analytic functions $f(z)$ of the complex variable $z$ carries over essentially intact to minimal surfaces. And, insofar as this principle applies to $\left|f^{\prime}(z)\right|$, in accordance with Theorem $B$ the principle largely carries over to the class of surfaces of nonpositive curvature. Since minimal surfaces are special surfaces of nonpositive curvature, the latter results hold in particular on minimal surfaces.

For example, we shall consider space analogues of the Lemma of Schwarz. These analogues might suggest various problems relative to the many ramifications and generalizations of the Lemma of Schwarz in complex variable theory. In particular, we note the result of Caratheodory [16] that the hypotheses of the Lemma of Schwarz imply $\left|f^{\prime}(z)\right| \leqq 1$ for $|z| \leqq 2^{1 / 2}-1$.

You will note that the Lemma of Schwarz applies to distances in the containing space, not to distances on the map $w=f(z)$. From Theorem A we can obtain the following result [7].

Let $S$,

$$
S: \quad x=x(u, v), \quad y=y(u, v), \quad z=z(u, v), \quad u^{2}+v^{2}<1,
$$


be a minimal surface given in conformal representation, such that $(0,0)$ is carried into $(0,0,0)$. If $S$ is comprised in the unit sphere, $x^{2}+y^{2}+z^{2}$ $\leqq 1$, then for $u^{2}+v^{2} \leqq r^{2}, 0<r<1$, we have

$$
[x(u, v)]^{2}+[y(u, v)]^{2}+[z(u, v)]^{2} \leqq r^{2} ;
$$

further, we have $\lambda(0,0) \leqq 1$. The signs of equality hold if and only if $S$ is a simply-covered circular disc with unit radius.

But now Theorem B suggests the possibility of an analogue of the Lemma of Schwarz for surfaces of nonpositive curvature involving distances on the surface $S$ itself [2]. We shall state the result only for the special case of a plane map.

Let $w=f(z)$ be analytic for $|z|<1$. If the length function

$$
l(r, \theta) \equiv \int_{0}^{r}\left|f^{\prime}\left(\rho e^{i \theta}\right)\right| d \rho
$$

satisfies

$$
l(r, \theta) \leqq 1
$$

for all $(r, \theta)$ with $0<r<1$, then we have

$$
l(r, \theta) \leqq r
$$

and

$$
\left|f^{\prime}(0)\right| \leqq 1,
$$

the signs of equality holding if and only if $f(z) \equiv e^{i \alpha} z$, where $\alpha$ is a real constant.

\section{REFERENCES}

1. E. F. Beckenbach, Generalized convex functions, Bull. Amer. Math. Soc. vol. 43 (1937) pp. 363-371.

2. - A relative of the Lemma of Schwarz, Bull. Amer. Math. Soc. vol. 44 (1938) pp. 698-707. 505.

3. ㄴ, An inequality of Jensen, Amer. Math. Monthly vol. 53 (1946) pp. 501-

4. - Some convexity properties of surfaces of negative curvature, to appear in Amer. Math. Monthly vol. 55 (1948).

5. E. F. Beckenbach and R. H. Bing, On generalized convex functions, Trans. Amer. Math. Soc. vol. 58 (1945) pp. 220-230.

6. E. F. Beckenbach, W. Gustin, and H. Shniad, On the mean modulus of an analytic function, to appear in Bull. Amer. Math. Soc.

7. E. F. Beckenbach and T. Rad6, Subharmonic functions and minimal surfaces, Trans. Amer. Math. Soc. vol. 35 (1933) pp. 648-661.

8. - Subharmonic functions and surfaces of negative curvature, Trans. Amer. Math. Soc. vol. 35 (1933) pp. 662-674. 
9. F. Bernstein and G. Doetsch, Zur Theorie der konvexen Funktionen, Math. Ann. vol. 76 (1915) pp. 514-526.

10. Bienaymé, Extraits des procès-verbaux des séances pendant l'année 1840, Société Philomatique de Paris, Paris, 1841, p. 67.

11. H. Blumberg, Convex functions, Trans. Amer. Math. Soc. vol. 20 (1919) pp. $40-44$.

12. T. Bonnesen, En bemaerkning om konvekse funktioner, Matematisk Tidsskrift B (1928) pp. 18-20.

13. T. Bonnesen and W. Fenchel, Theorie der konvexen Körper, Berlin, Springer, 1934.

14. H. Busemann, Spaces with non-positive curvature, to appear in Acta. Math. (1948).

15. C. Carathéodory, On Dirichlet's problem, Amer. J. Math. vol. 59 (1937) pp. 709-731.

16. - Eine Verschärfung des Schwarzschen Lemmas, Praktika Akademias Athenōn, vol. 11 (1936) pp. 276-286.

17. T. Carleman, Zur Theorie der Minimalfächen, Math. Zeit. vol. 9 (1921) pp. 154-160.

18. E. Cartan, Leçons sur la géométrie des surfaces de Riemann, Paris, GauthierVillars, 1928.

19. A. L. Cauchy, Cours d'analyse de l'Ecole Royale Polytechnique, part 1, Analyse algebrique, Paris, 1821.

20. M. Drandell, On generalized convex functions, not yet published.

21. G. Faber, Über die zusammengehörigen Konvergenzradien von Potenzreihen mehrerer Verändlicher, Math. Ann. vol. 61 (1905) pp. 289-324.

22. E. Fabry, Sur les rayons de convergence d'une série double, C. R. Acad. Sci. Paris vol. 134 (1902) pp. 1190-1192.

23. J. W. Green and W. Gustin, Subconvex sets, not yet published.

24. J. Hadamard, Etude sur les proprietes des fonctions entières et en particulier d'une fonction considérée par Riemann, J. Math. Pures Appl. vol. 58 (1893) pp. 171215.

25. - Sur les fonctions entieres, Bull. Soc. Math. France vol. 24 (1896) pp. $186-187$.

26. - Sur certaines propriêtés des trajectoires en dynamique, J. Math. Pures Appl. vol. 62 (1897) pp. 331-388.

27. - Les surfaces a courbures opposées et leurs lignes géodésiques, J. Math. Pures Appl. vol. 63 (1898) pp. 27-73.

28. G. Hamel, Eine Basis aller Zahlen und die unstetigen Lösungen der Funktionalgleichung: $f(x)+f(y)=f(x+y)$, Math. Ann. vol. 60 (1905) pp. 459-462.

29. G. H. Hardy, The mean value of the modulus of an analytic function, Proc. London Math. Soc. (2) vol. 14 (1915) pp. 269-277.

30. G. H. Hardy, J. E. Littlewood, G. Polya, Inequalities, Cambridge, England, University Press, 1934.

31. F. Hartogs, Zur Theorie der analytischen Funktionen mehrerer unabhängiger Veränderlicher, insbesonderere ïber die Darstellung derselben durch Reihen, welche nach Potenzen einer Veränderlichen fortschreiten, Math. Ann. vol. 62 (1906) pp. 1-88.

32. M. O. Hölder, Über einen Mittelwertsatz, Nachr. Ges. Wiss. Göttingen, 1889, pp. 38-47.

33. J. L. W. V. Jensen, Sur un nouvel et important theorème de la theorie des fonctions, Acta Math. vol. 22 (1898-1899) pp. 359-364. 
34. - Om konvexe Funktioner og Uligheder mellem Middelvaerdier, Nyt. Tidsskrift for Mathematik vol. 16 B (1905) pp. 49-69.

35. - - Sur les fonctions convexes et les inegalites entre les valeurs moyennes, Acta Math. vol. 30 (1906) pp. 175-193.

36. F. B. Jones, Connected and disconnected plane sets and the functional equation $f(x)+f(y)=f(x+y)$, Bull. Amer. Math. Soc. vol. 48 (1942) pp. 115-120.

37. G. Julia, Sur les familles de fonctions analytiques de plusieurs variables, Acta Math. vol. 47 (1926) pp. 53-115.

38. - Sur les moyennes des modules de fonctions analytiques, Bull. Sci. Math. (2) vol. 51 (1927) pp. 198-214.

39. - Principes géometriques d'analyse, part 2, Paris, Gauthier-Villars, 1932.

40. E. Landau, Darstellung und Begrilndung einiger neuerer Ergebnisse der Funktionentheorie, Berlin, Springer, 1916.

41. E. E. Levi, Studii sui punti singolari essenziali delle funzioni analitiche di due o piu variabili complesse, Annali di Matematica Pura ed Applicata (3) vol. 17 (1910) pp. 61-87.

42. E. J. McShane, On a certain inequality of Steiner, Ann. of Math. (2) vol. 33 (1932) pp. 125-138. 527.

43. —, Jensen's inequality, Bull. Amer. Math. Soc. vol. 43 (1937) pp. 521-

44. H. Minkowski, Volumen und Oberfläche, Math. Ann. vol. 57 (1903) pp. 447495.

45. P. Montel, Sur les fonctions convexes et les fonctions sousharmoniques, J. Math. Pures Appl. (9) vol. 7 (1928) pp. 29-60.

46. Z. Nehari, Une proprieté des valeurs moyennes d'une fonction analytique, C. R. Acad. Sci. Paris vol. 208 (1939) pp. 1785-1787.

47. R. Nevanlinna, Zur Theorie der meromorphen Funktionen, Acta Math. vol. 46 (1925) pp. 1-99.

48. N. Norris, Convexity properties of generalized mean value functions, Ann. Math. Statist. vol. 8 (1937) pp. 118-120.

49. A. Ostrowski, Zur Theorie der konvexen Funktionen, Comment. Math. Helvetici vol. 1 (1929) pp. 157-159.

50. O. Perron, Eine neue Behandlung der ersten Randwertaufgabe für $\Delta u=0$, Math. Zeit. vol. 18 (1923) pp. 42-54.

51. E.Phragmén and E. Lindelöf, Sur une extension d'un principe classique de l'analyse et sur quelques proprietes de fonctions monogenes dans le voisinage d'un point singulier, Acta Math. vol. 31 (1908) pp. 381-406.

52. H. Poincaré, Sur le problème de la distribution electrique, C. R. Acad. Sci. Paris vol. 104 (1887) p. 44.

53. G. Pólya, Untersuchungen über Liicken und Singularitäten von Potenzreihen, Math. Zeit. vol. 29 (1929) pp. 549-560.

54. G. Pólya and G. Szegö, Aufgaben und Lehrsätze aus der Analysis, Berlin, Springer, 1925.

55. T. Popoviciu, Les fonctions convexes, Paris, Hermann, 1945.

56. A. Pringsheim, Zur Theorie der ganzen tranzendenten Funktionen (Nachtrag), Münchner Sitzungsberichte vol. 32 (1902) pp. 295-304.

57. T. Rad6, On convex functions, Trans. Amer. Math. Soc. vol. 37 (1935) pp. 266285.

58. —-, Subharmonic functions, Berlin, Springer, 1937. 
59. T. Rad6 and F. Riesz, Über die erste Randwertaufgabe für $\Delta u=0$, Math. Zeit. vol. 22 (1925) pp. 41-44.

60. R. Remak, Über potentialkonvexe Funktionen, Math. Zeit. vol. 20 (1924) pp. 126-130.

61. F. Riesz, Sur les valeurs moyennes du module des fonctions harmoniques et des fonctions analytiques, Acta Univ. Szeged. vol. 1 (1922) pp. 27-32.

62. - Über subharmonische Funktionen und ihre Anwendung in der Funktionentheorie, Jber. Deutschen Math. Verein. vol. 33 (1925) pp. 86-87.

63. - Über subharmonische Funktionen und ihre Rolle in der Funktionentheorie und in der Potentialtheorie, Acta Univ. Szeged. vol. 2 (1925) pp. 87-100.

64. - - Sur les fonctions subharmoniques et leur rapport a la théorie du potentiel, Acta Math. vol. 48 (1926) pp. 329-343, and vol. 54 (1930) pp. 321-360.

65. _- Su alcune disuguaglianze, Boll. dell'Unione Matematica Italiana vol. 7 (1928) pp. 77-79.

66. H. Shniad, On the convexity of mean value functions, to appear in Bull. Amer. Math. Soc. vol. 54 (1948).

67. W. Sierpinski, Sur les fonctions convexes mesurables, Fund. Math. vol. 1 (1920) pp. 125-129.

68. O. Stolz, Grundzüge der Differential- und Integralrechnung, vol. I, Leipzig, Teubner, 1893.

69. G. Valiron, Fonctions convexes et fonctions entières, Bull. Soc. Math. France, vol. 60 (1932) pp. 278-287.

70. A. Weil, Sur les surfaces a courbure negative, C. R. Acad. Sci. Paris vol. 182 (1926) pp. 1069-1071.

71. H. Whitney, Note on Perron's solution of the Dirichlet problem, Proc. Nat. Acad. Sci. U. S. A. vol. 18 (1932) pp. 68-70.

72. N. Wiener, Note on a paper of O. Perron, Journal of Mathematics and Physics, Massachusetts Institute of Technology, vol. 4 (1925) pp. 21-32.

University of California at Los ANgeles 